\title{
Lipopolysaccharide Alters Motivated Behavior in a Monetary Reward Task: a Randomized Trial
}

\author{
Julie Lasselin *, I,2,3, Michael T Treadway ${ }^{4}$, Tamara E Lacourt ${ }^{5}$, Anne Soop ${ }^{6}$, Mats J Olsson', Bianka Karshikoff ${ }^{1,2}$, \\ Sofie Paues-Göranson ${ }^{6}$, John Axelsson ${ }^{1,2,7}$, Robert Dantzer ${ }^{5}$ and Mats Lekander ${ }^{1,2,7}$ \\ 'Division for Psychology, Department of Clinical Neuroscience, Karolinska Institutet, Stockholm, Sweden; ${ }^{2}$ Stress Research Institute, Stockholm \\ University, Stockholm, Sweden; ${ }^{3}$ Institute of Medical Psychology and Behavioral Immunobiology, Universitätsklinikum Essen, Essen, Germany; \\ ${ }^{4}$ Department of Psychology, Emory University, Atlanta, GA, USA; ${ }^{5}$ Division of Internal Medicine, Department of Symptom Research, The University \\ of Texas, MD Anderson Cancer Center, Houston, TX, USA; 'Department of Clinical Sciences, Danderyd Hospital, Karolinska Institutet, Stockholm, \\ Sweden; ${ }^{7}$ Osher Center for Integrative Medicine, Karolinska Institutet, Stockholm, Sweden
}

Inflammation-induced sickness is associated with a large set of behavioral alterations; however, its motivational aspects remain poorly explored in humans. The present study assessed the effect of lipopolysaccharide (LPS) administration at a dose of $2 \mathrm{ng} / \mathrm{kg}$ of body weight on motivation in 21 healthy human subjects in a double-blinded, placebo (saline)-controlled, cross-over design. Incentive motivation and reward sensitivity were measured using the Effort Expenditure for Rewards Task (EEfRT), in which motivation for high-effort/high-reward trials vs low-effort/low-reward trials are manipulated by variations in reward magnitude and probability to win. Because of the strong interactions between sleepiness and motivation, the role of sleepiness was also determined. As expected, the probability to win predicted the choice to engage in high-effort/high-reward trials; however, this occurred at a greater extent after LPS than after saline administration. This effect was related to the level of sleepiness. Sleepiness increased motivation to choose the high-effort/high-reward mode of response, but only when the probability to win was the highest. LPS had no effect on reward sensitivity either directly or via sleepiness. These results indicate that systemic inflammation induced by LPS administration causes motivational changes in young healthy subjects, which are associated with sleepiness. Thus, despite its association with energy-saving behaviors, sickness allows increased incentive motivation when the effort is deemed worthwhile.

Neuropsychopharmacology (2017) 42, $801-81$ 0; doi:10.1038/npp.2016.191; published online 12 October 2016

\section{INTRODUCTION}

Systemic inflammation is associated with a series of behavioral alterations, called 'sickness behavior' (Dantzer et al, 2008). Sickness behavior is characterized by alterations in motivational priorities, as fighting pathogens and taking care of the sick body predominate over other homeostatic and reproductive goals (Aubert, 1999). Motivated behavior depends on sensitivity to reward (or pleasure) and incentive motivation (ie, willingness to expend effort in order to obtain a reward; Berridge et al, 2009). Animal studies have shown apparently decreased sensitivity to reward or anhedonia during immune activation, such as after administration of lipopolysaccharide (LPS; Aubert, 1999; Larson, 2002). Additional preclinical studies indicate, however, that inflammation-induced motivational changes are characterized by a reduction in incentive motivation, rather than a decrease in sensitivity to reward (Anisman et al, 1998; De La

*Correspondence: Dr J Lasselin, Institute of Medical Psychology and Behavioral Immunobiology, Universitätsklinikum Essen, Hufelandstrasse 55, Essen 45 122, Germany, Tel: +49 201723 476I, Fax: +49 201723 5948, E-mail: julie.lasselin@uk-essen.de

Received 5 August 2016; accepted I September 20 16; accepted article preview online 13 September 2016
Garza et al, 2005; Merali et al, 2003; Nunes et al, 2014; Vichaya et al, 2014). These observed changes in motivation can result from changes in striatal dopamine function (Miller and Raison, 2015) that has a critical role in willingness to expend effort for reward in effort-based decision-making paradigms (Salamone et al, 2007; Wardle et al, 2011). Clinical studies report attenuated striatal response to rewarding outcomes after an inflammatory stimulus (Capuron et al, 2012; Eisenberger et al, 2010; Felger and Miller, 2012). Despite all these data, the motivational alterations that are caused by the activation of the immune system have not been characterized in humans.

At the subjective level, fatigue and sleepiness are integral components of the sickness response (Dantzer et al, 2014; Harrison et al, 2009; Hermann et al, 1998), acting as strong motivational signals supporting rest and recovery. Hence, they are probably related to the motivational alterations that are associated with inflammation. The objective of the present study was therefore to characterize how inflammation has an impact on motivated behavior and to assess whether sleepiness, which can be easily and reliably measured with the Karolinska Sleepiness Scale (KSS; Akerstedt et al, 2014), plays a role in the effects of inflammation. LPS was used to induce inflammation and 
motivation was assessed using the Effort Expenditure for Rewards Task (EEfRT; Treadway et al, 2009). The EEfRT was originally designed as a human analog to rodent effort-based decision-making paradigms, allowing the comparison of the outcomes obtained in human studies with those from rodent studies. In addition, this task contrasts a high-effort/highreward mode of response to a low-effort/low-reward mode of response, together with variations in the probability and magnitude of monetary rewards. This aspect is close to natural conditions, in which choices between different options are common. This task also allows differentiating incentive motivation (ie, willingness to expend effort in order to obtain a reward) from sensitivity to reward. For instance, depressed patients display a decreased willingness to expend effort for reward, in particular with respect to the high-effort/high-reward trials, combined with a weaker effect of reward values on the choice for high-effort/highreward trials (ie, reduced sensitivity to reward cues; Treadway et al, 2012).

On the basis of mouse studies using a similar task (Vichaya et al, 2014), we hypothesized that LPS administration would reduce incentive motivation, measured by a decrease in number of choices of high-effort/high-reward trials. We predicted that this effect would be stronger at lower levels of probability to win (ie, when the energy expenditure was less worthwhile). We also expected that the sensitivity to reward would not be affected, ie, that increase in reward magnitude would predict more choices of high-effort/high-reward trials in a similar way after exposure to LPS or saline. In order to determine whether the potential effect of LPS on reward motivation was related to sleepiness, we assessed the following: (1) the effect of LPS administration on reward motivation in the EEfRT; (2) the effect of sleepiness on reward motivation; and (3) the combined effect of LPS administration and sleepiness on reward motivation.

\section{MATERIALS AND METHODS}

\section{Participants}

Healthy volunteers were recruited through advertisements posted at university campuses. Eligibility criteria included the following: age 18-50 years, no known physiological or psychiatric disease, non-smoker, no excessive alcohol use, and body mass index $18.5-29 \mathrm{~kg} / \mathrm{m}^{2}$. Each participant underwent a medical examination by a physician. The absence of major depressive disorder was verified using the M.I.N.I. International Neuropsychiatric Interview (Sheehan et al, 1998) In addition, clinical laboratory analyses (ie, level of sodium, potassium, creatinine, transaminases, white blood cell count, and hemoglobin) were performed before the inclusion to eliminate any possible medical condition.

Twenty-two volunteers (mean age: $23 \pm 4$; nine women) were included in the protocol. The sample size was determined according to previous studies, showing that a sample size of $n=20-25$ is sufficient to measure significant immunological and behavioral changes after LPS administration (Calvano and Coyle, 2012; Hallstrom et al, 2011). None of the subjects dropped out from the study; however, one subject could not complete any of the high-effort tasks in either condition and was therefore removed from the analyses. Remuneration for participation was 3500 SEK
( $\sim 370 €)$. Participants were told that they would receive 3000 SEK for study participation and a bonus of up to 500 SEK depending on how much they would win in the EEfRT (which was presented to them as a 'computer game'); however, all subjects received the 500 SEK bonus remuneration at the end of the study.

The study was conducted according to ethical standards and was approved by the regional ethical review board in Stockholm, Sweden (Dnr 2015/1415-32; ClinicalTrials.gov identifier: NCT02529592). All participants signed a written informed consent after a complete explanation of the study.

\section{LPS Administration Protocol}

The protocol was conducted in the Center for Clinical Research at Danderyd Hospital, Stockholm, Sweden in February to April 2015. A double-blinded, placebo-controlled, cross-over design was used. The subjects were randomly allocated by the physician (simple randomization using sealed envelopes) to receive either an LPS injection (Escherichia coli endotoxin, Lot HOK354, CAT number 1235503, United States Pharmacopeia, Rockville, MD, USA) of $2 \mathrm{ng} / \mathrm{kg}$ body weight (dissolved in $0.9 \% \mathrm{NaCl}$ ) or the same volume of physiological saline $(0.9 \% \mathrm{NaCl})$ on the first session of the study, between 0830-0930 hours. After 34 weeks of wash-out, allowing the elimination of residual effects of LPS administration and women to be in a similar menstrual cycle phase, the second session occurred in which subjects were assigned the reverse treatment.

All volunteers and research staff were blinded, except the physician involved in the study for security purposes.

\section{Body Temperature, Cytokine Concentrations, and Sickness Symptoms}

Tympanic temperature was measured before and $0.5,1,1.5$, $2,2.5,3,3.5,4,5$, and $7 \mathrm{~h}$ after the injection.

Blood samples were drawn before the injection and 1, 1.5, 2, 3, 4, 5, and $7 \mathrm{~h}$ after the injection. Cytokine plasma concentrations of the pro-inflammatory cytokines, interleukin (IL)-6, tumor necrosis factor- $\alpha$, and IL-8, were assessed using high-sensitivity multiplex (Human Mag Luminex Performance Assay, LHSCM000, LHSCM206, LHSCM208, and LHSCM210, RnD Systems, MN, USA). Logarithmtransformed concentrations of IL- 6 are reported in the current study as an index of cytokine production. Four blood samples were missed because of technical constraints (two in each condition). The average of the two values of the time points just before and just after, or the value just after (when baseline value was missing), was then used.

The presence and intensity of headaches and nausea were assessed by the caregiver at the same time points that body temperature on a scale ranging from 0 (not at all) to 10 (very intense). In addition, sickness symptoms were assessed using the Sickness Questionnaire (SicknessQ) before, 1.5, 3, 5, and $7-7.5 \mathrm{~h}$ after the injection. The SicknessQ comprises 10 items that assess several aspects of sickness at the time when the questionnaire is completed with a total score ranging from 0 (no symptom) to 30 (very high sickness symptoms; Andreasson et al, 2016). The SicknessQ was completed by the subject except $1.5 \mathrm{~h}$ after the injection, when the caregiver 
conducted the questionnaire (because of some subjects' weakness).

\section{Sleepiness}

Subjective sleepiness was assessed with the KSS (Akerstedt et al, 2014). The KSS measures the level of sleepiness that the subject feels 'right now', on a 9-point scale from $1=$ extremely alert to $9=$ very sleepy, fighting sleep, and need effort to keep awake. The KSS was completed by the subjects before, 3 and $7 \mathrm{~h}$ after the LPS/saline administration.

\section{State Anxiety, Positive Affect, and Vigor}

Anxiety and positive affect were measured in order to adjust the effect of sleepiness for mood state. Anxiety was assessed using the State version of the State-Trait Anxiety Inventory (STAI; Spielberger et al, 1979). Positive affect was assessed using the valence dimension of the Swedish Core Affect Scale (SCAS; Vastfjall and Garling, 2007). In addition, in order to evaluate the association of sleepiness with another, overall, measurement of fatigue, vigor was measured using the activation dimension of the SCAS (Vastfjall and Garling, 2007).

The STAI and SCAS questionnaires were completed by the subjects before, 3 and $7 \mathrm{~h}$ after the LPS/saline administration.

\section{EEfRT}

In the EEfRT, subjects repeatedly completed tasks in a timespan of $20 \mathrm{~min}$ in order to win monetary rewards (Treadway et al, 2009). Subjects had to choose between easy or hard tasks, which were associated with different reward levels and needed to be completed in order to win the reward. In order to complete the easy (low-effort/low-reward) task, the subjects had to make 30 button presses in $7 \mathrm{~s}$ using the index finger of the dominant hand. In the hard (high-effort/highreward) task, the subjects had to make 100 button presses in $21 \mathrm{~s}$ using the little finger of the non-dominant hand.

The monetary reward for the low-effort/low-reward trial was always $1 \$$. The reward for the high-effort/high-reward trial was higher and varied randomly per trial between $\$ 1.24$ and $\$ 4.21$. In addition, a probability contingency was added $(12,50$, and $88 \%)$ so that, even if they completed the trial, subjects won the monetary reward only on 12,50 , or $88 \%$ of the cases. The reward magnitude for the high-effort/highreward trial and the probability of winning were presented at the beginning of each trial (see Supplementary Figure S1A). After choosing between the low-effort/low-reward and the high-effort/high-reward task, the subjects completed the task by pressing the respective button with the respective finger (Supplementary Figure S1B). After the trial, the subjects were informed whether they succeeded in the task and whether they won the money reward (Supplementary Figure S1C and D). The main outcome of the EEfRT task was the choice of the high-effort/high-reward mode of response. Number of high-effort trial choices in the test represented the level of effort subjects were willing to employ in order to get a higher reward (ie, incentive motivation). The effects of reward magnitude and probability in predicting high-effort choices were also assessed.

To ensure monetary reward motivation, the subjects were told that four trials would be randomly selected among the successful trials, and the total amount won in these four trials would be used to calculate their bonus remuneration (up to 500 SEK) in a proportional way. They were also told that any cheating (eg, using a different finger to complete the task) would be punished by not getting any reward.

The EEfRT was conducted between 4 and $5 \mathrm{~h}$ after the LPS/saline injection, when sickness was still present in LPS-treated subjects but more severe effects (eg, shivering, severe nausea, and headache) had subsided. Preliminary experiments indicated that the task should not be conducted earlier (eg, between 3 and $4 \mathrm{~h}$ after the injection, as originally planned) as subjects were then usually too weak to perform this task.

\section{Statistical Analyses}

All the subjects completed the monetary reward task on each study day. However, as stated above, one subject could not complete any of the high-effort tasks in either condition and was therefore removed from the analyses. In addition, EEfRT data from one subject in the LPS condition and from two subjects in the saline condition were unavailable because of technical problems. This means that 21 subjects for whom data were available for at least one condition were included in the analyses of the present study. In addition, two subjects were outliers (value higher than mean $+3 \mathrm{SD}$ ) with respect to at least two inflammatory markers (baseline concentrations of one subject and peak concentrations after LPS administration of another subject). These two subjects were therefore excluded from the analyses on IL- 6 data.

The effect of treatment on body temperature, plasma IL-6 concentrations, and sickness symptoms (headache, nausea, and SicknessQ-score) from baseline to $7 \mathrm{~h}$ after the injection was assessed using repeated measurements (RM) ANOVA with treatment (LPS/saline) and time as independent variables and post hoc pairwise comparisons to assess the effect of condition at each time point. The effect of treatment on sleepiness, state anxiety, positive affect, and vigor was assessed in the same way.

The effect of treatment on performance in the EEfRT (ie, proportion of successful trials, time to completion, and button press rate) was analyzed using RM ANOVA with treatment (LPS/saline) as independent variable.

Predictive effects of LPS vs saline treatment and sleepiness on high-effort/high-reward trial choices were tested with generalized estimating equation (GEE) using a binary logistic model. Within-subject variables were study day (first/second session), trial of the EEfRT, reward magnitude, and probability levels, using an exchangeable working correlation structure. The effects of condition and sleepiness on the choice of the high-effort/high-reward trial (no/yes), and the statistical mediation effect of sleepiness on the association between condition and high-effort/high-reward choices were assessed in three separate models: (1) the first model assessed the effect of condition (LPS/saline) and its interaction with probability levels (continuous variable) and reward magnitude (continuous variable); (2) the second model assessed the effect of sleepiness (KSS scores $3 \mathrm{~h}$ after LPS/saline administration) and its interaction with probability levels and reward magnitude; (3) the third model assessed the effect of condition and sleepiness together and their interaction with probability levels and reward magnitude. In order to adjust 
for mood state and sickness symptoms, state anxiety (STAI), positive affect (SCAS valence), and sickness (SicknessQ) scores measured $3 \mathrm{~h}$ after the LPS/saline administration were added in the models as covariates. These analyses were repeated entering the order of the LPS injection (on first $v s$ second study day) in order to control for a contaminating effect of a potential unblinding. The indirect effect of sleepiness was then confirmed using the PROCESS macro (http://www.processmacro.org/) developed by Hayes (2013) using average proportions of high-effort/high-reward task choices. These analyses were repeated using the peak concentration of IL-6 (ie, individual highest logarithm concentration) observed between baseline and $5 \mathrm{~h}$ after the LPS/saline injection instead of treatment as independent variable to confirm that the effects of LPS administration could be attributed to inflammation. The peak of IL-6 concentration was used instead of a fixed time point (eg, at the time of the EEfRT) as it represents the largest effect of LPS administration and reflects how much cytokines would have had an impact on the brain.

When significant interaction with reward magnitude or probability levels was found, exploratory post hoc GEE analyses were performed to investigate at which level of probability $(12,50$, and $88 \%)$ or size of the monetary reward (low: $<2 \$$, medium: $2-3 \$$, and high: $>3 \$$ ) the respective effect appeared.

All statistical analyses were performed using IBM SPSS statistics 22 with a degree of significance set at $P<0.05$.

\section{RESULTS}

\section{Validation of the LPS Administration Model}

LPS, but not saline, administration led to significant increases in body temperature, IL- 6 concentrations, and sickness symptoms (Figure 1, see Supplementary Table S1 for detailed statistics). In particular, the increase in body temperature and IL- 6 concentrations was observed between 1 and $3 \mathrm{~h}$ after the LPS injection. The effects were stronger than what has been previously reported in studies using lower dose of LPS from the same lot (eg, $0.8 \mathrm{ng} / \mathrm{kg}$ bw; see, for instance, Engler et al, 2016) but similar to what has been found in studies using a dose of $2 \mathrm{ng} / \mathrm{kg}$ bw from a different lot (eg, Suffredini et al, 1999; Calvano and Coyle, 2012). Headache and nausea severity peaked $1.5 \mathrm{~h}$ after LPS injection, and high levels of sickness symptoms were measured 1.5 and $3 \mathrm{~h}$ after the injection. The monetary reward task was performed in the descending phase of the body temperature, cytokine, and sickness responses, but when the effects of LPS administration were still present (4-5 $\mathrm{h}$ after the injection; Figure 1).

\section{Effect of LPS Administration on Sleepiness, State Anxiety, Positive Affect, and Vigor}

Sleepiness was affected by time $(P<0.001)$ and condition $(P=0.045)$, and a trend for an interaction between time and condition $(P=0.08)$ was found (for detailed statistics, see Supplementary Table S1). Sleepiness was significantly increased $3 \mathrm{~h}$ after LPS in comparison with saline administration (Figure 1).
State anxiety, positive affect, and vigor were affected by time and condition, indicated by significant time $x$ condition interactions (Supplementary Table S1). State anxiety increased while positive affect and vigor decreased after LPS, but not saline, administration (Figure 1). The increase in state anxiety was slightly higher than what was observed in previous studies using a lower dose of LPS from the same or different lot (eg, Engler et al, 2016; Karshikoff et al, 2015; Lasselin et al, 2016).

Three hours after LPS administration, increased sleepiness was inversely correlated with vigor $(r=-0.510, P=0.018)$ and positive affect (LPS: $r=-0.494, P=0.023$ ).

\section{Effects of LPS on EEfRT Performance}

Compared with saline injection, LPS-treated subjects had a reduced success rate in the low-effort trials $(\mathrm{F}(1,17)=4.5$, $\left.\eta^{2}=0.21, P=0.049\right)$ but performed better when engaged in high-effort trials $\left(\mathrm{F}(1,17)=7.9, \eta^{2}=32, P=0.012\right.$; Figure $\left.2 \mathrm{a}\right)$. They were also faster in completing the high-effort trials $\left(\mathrm{F}(1,17)=8.0, \eta^{2}=0.32, P=0.011\right)$, and this was due to an increased button press rate $\left(\mathrm{F}(1,17)=12.1, \quad \eta^{2}=0.42\right.$, $P=0.003$; Figure $2 b)$. No such difference was found in the low-effort trials $\left(\eta^{2}=0.12, P=0.15\right.$ and $\eta^{2}=0.10, P=0.18$; Figure 2b). These results indicate that LPS enhanced behavioral performance in high-effort/high-reward trials.

\section{Effect of LPS and Sleepiness on High-Effort/High- Reward Choices}

Table 1 shows the results of the GEE models assessing the effects of (1) condition (model 1), (2) sleepiness (model 2), (3) condition and sleepiness together (model 3) on the choice of high-effort/high-reward trial (yes/no). Interactions with probability levels and reward magnitude on high-effort/high-reward choices are also displayed.

The three models show a negative effect of trial order, indicating that subjects made overall less high-effort/highreward choices at the end of the task in comparison with the beginning. In addition, increase in reward magnitude and in probability levels significantly predicted more high-effort/high-reward choices. This means that the participants were more likely to engage in high-effort/highreward trials when the reward or the probability to win were higher.

In the first model (Table 1), there was a significant positive interaction between LPS administration and probability to win. The direction of the interaction indicates a greater association between increased probability levels and higheffort/high-reward choices in the LPS condition in comparison with saline administration. In other words, in comparison with the saline condition, LPS-treated subjects were more likely to choose the high-effort/high-reward trials when the probability to win was higher (Figure 3a). Similar associations were found when entering peak IL- 6 concentration instead of condition as independent variable in the model (peak IL-6 $\times$ probability, $B=0.006(0.003), P=0.036$; see Supplementary Table S2, GEE model 1 for detailed statistics).

When assessing the effect of sleepiness, a similar interaction effect as in the first model was found with probability levels (model 2 in Table 1). This indicates that the more 
sleepy the subjects felt, the more likely they were to engage in high-effort/high-reward trials when the probability level was higher (Figure 3b).
When assessing the effect of LPS administration and sleepiness together (model 3 in Table 1), the interaction between LPS and probability was no longer significant,
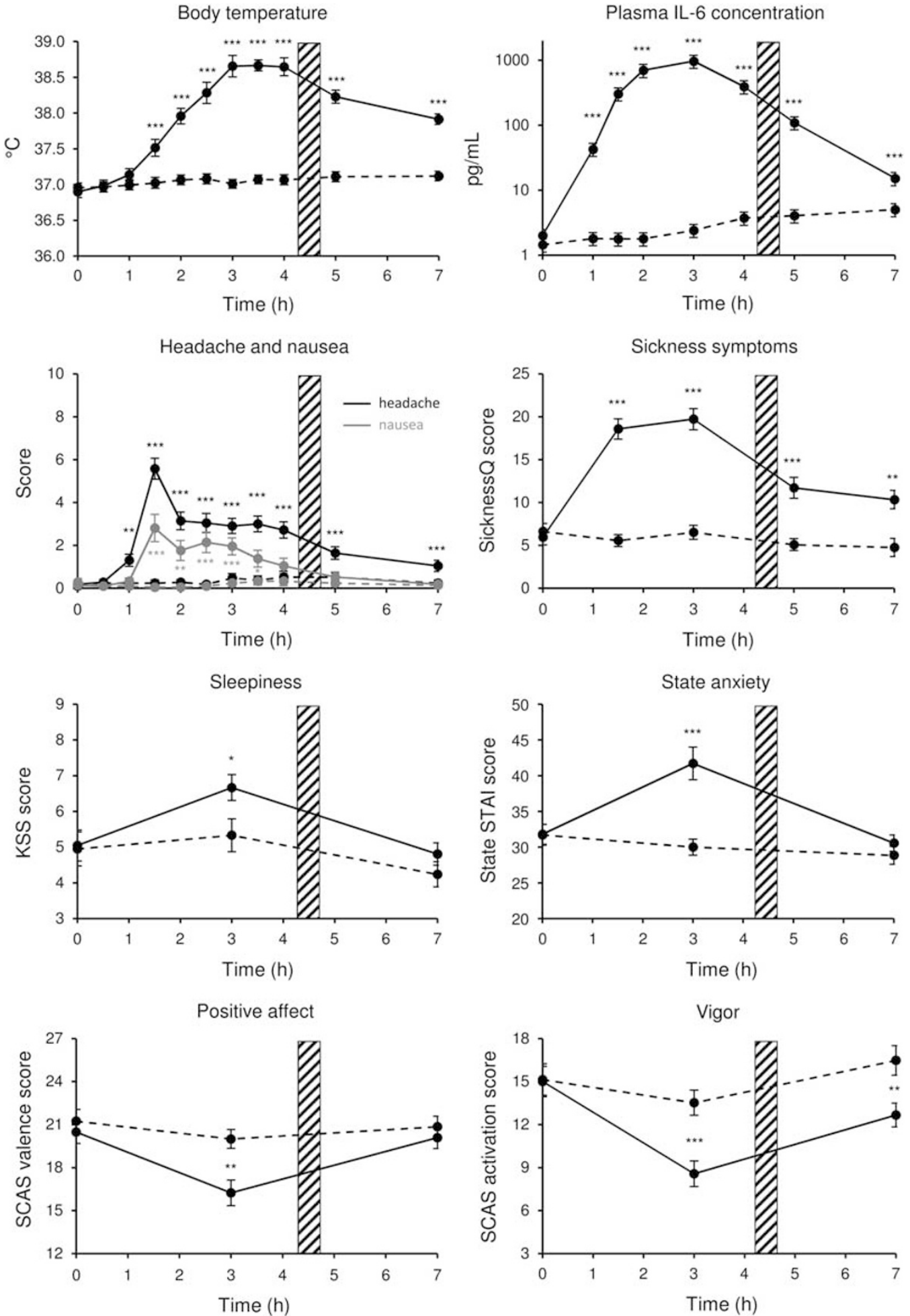

Figure I Effect of lipopolysaccharide (LPS) administration in comparison with saline administration. LPS administration (plain lines) significantly induced an increase in body temperature, plasma interleukin-6 (IL-6) log-transformed concentrations as well as sickness symptoms such as headache, nausea or overall sickness symptoms, in comparison to saline administration (dashed lines). Sleepiness and state anxiety were increased, while positive affect and vigor was decreased, 3h after LPS administration in comparison to saline administration. The monetary reward task (EEfRT) was performed between 4h and 5h after LPS/saline injection (shaded area), when sickness was still present in LPS-treated subjects but when the more severe effects had subsided. The Effort Expenditure for Rewards Task (EEfRT) lasted 20 min. **** $P<0.00$ I, ** $P<0.01$, *P $<0.05$ LPS vs saline condition. 
a
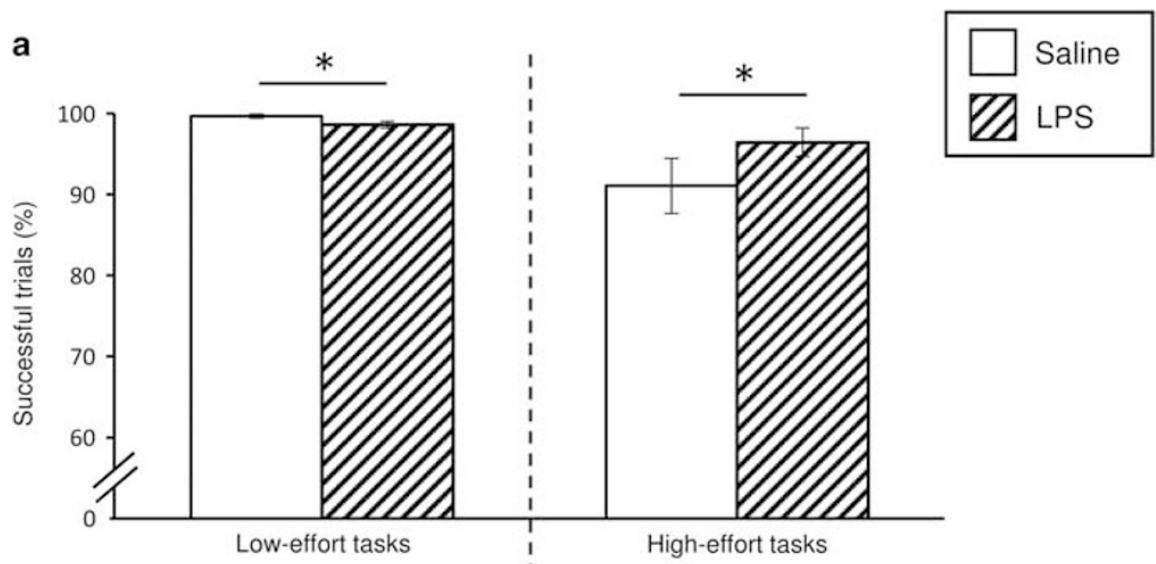

b

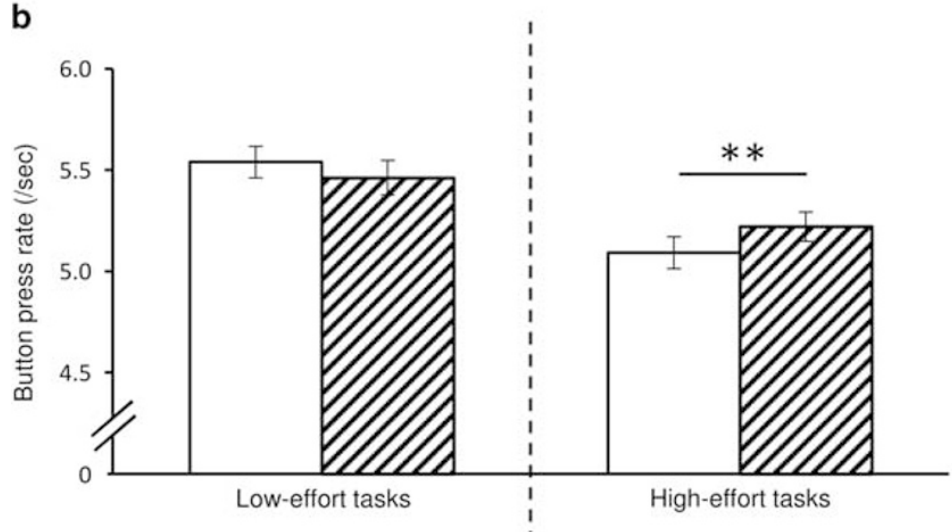

Figure 2 Effect of lipopolysaccharide (LPS) vs saline administration on performance in the low-effort and high-effort trials of the Effort Expenditure for Rewards Task (EEfRT). (a) Proportion of successful trials and (b) the mean button press rate per second in the low-effort and in the high-effort trials of the EEfRT, after LPS vs saline administration. Errors bars are SEM. $* P<0.05$; $* * P<0.01$.

Table I Effect of LPS vs Saline Administration and Sleepiness on High-Effort/High-Reward Task Choices in the EEfRT

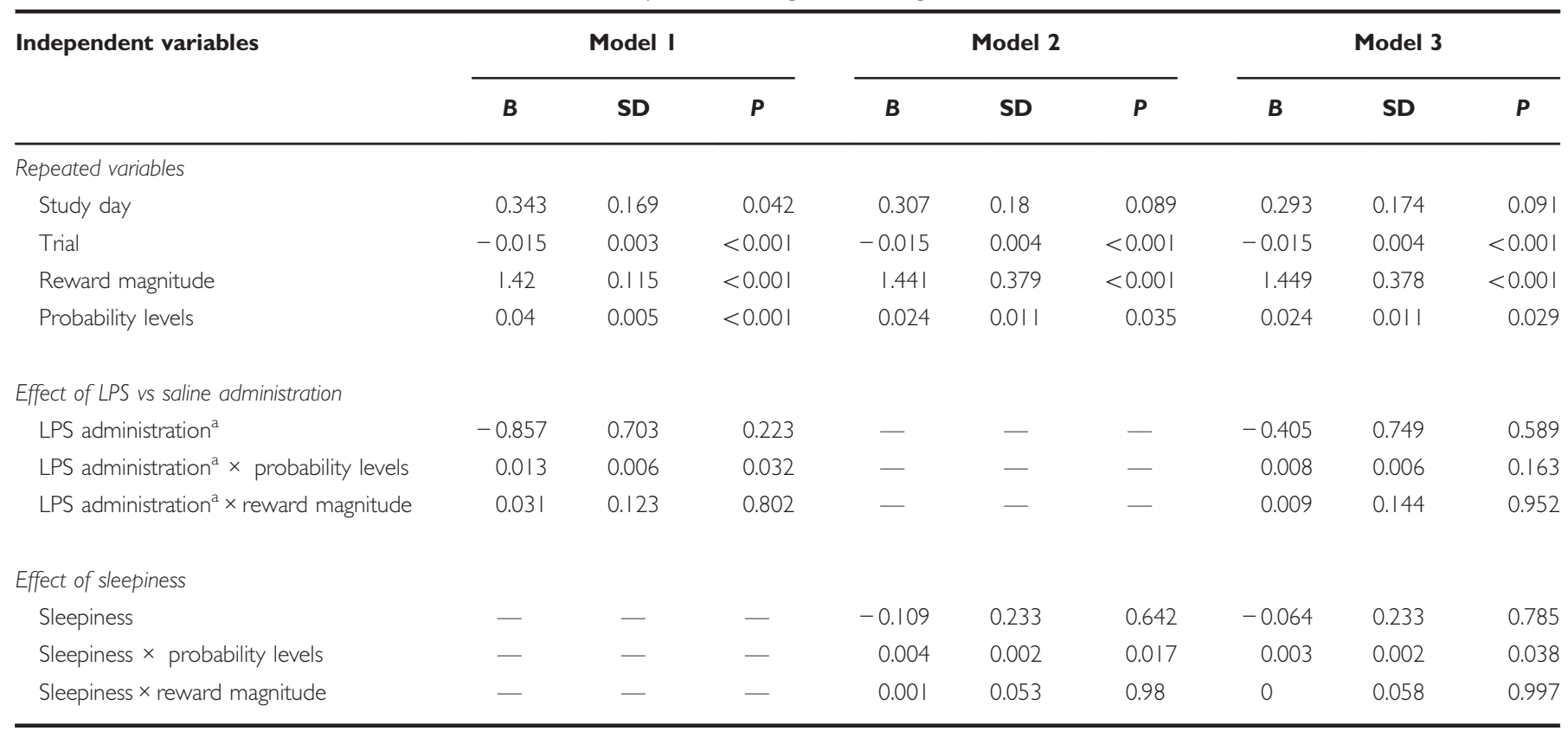

Generalized estimating equation (GEE) using a binary logistic model and high-effort task choice (no/yes) as dependent variable and adjusted for mood state (state anxiety and positive affect) and sickness symptoms (sickness Q scores). Model I assesses the effect of LPS vs saline administration and its interaction with probability levels and reward magnitude. Model 2 assesses the effect of sleepiness (measured in both LPS and saline conditions) and its interaction with probability levels and reward magnitude. Model 3 assesses the effect of LPS vs saline administration and sleepiness together, and their interaction with probability levels and reward magnitude. a $\mathrm{S} a$ line is the condition of reference. 

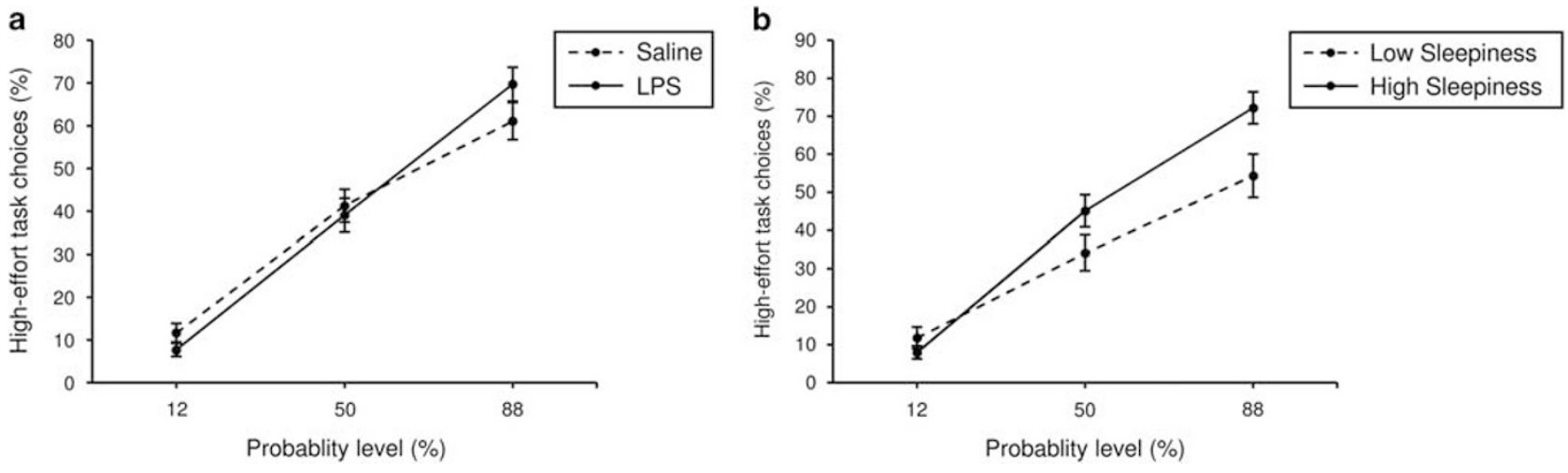

Figure 3 Effect of probability levels on high-effort/high-reward choices in the Effort Expenditure for Rewards Task (EEfRT). Proportion of high-effort/highreward choices depending on each probability level (a) after lipopolysaccharide (LPS) vs saline administration and (b) in subjects with low (ie, < first tertile, 5) and high (ie, $\geq$ second tertile, 7) levels of sleepiness. Errors bars are SEM.

Table 2 Post hoc Analyses of the Effect of Sleepiness on High-Effort/High-Reward Task Choices at Each Level of Probability of the EEfRT

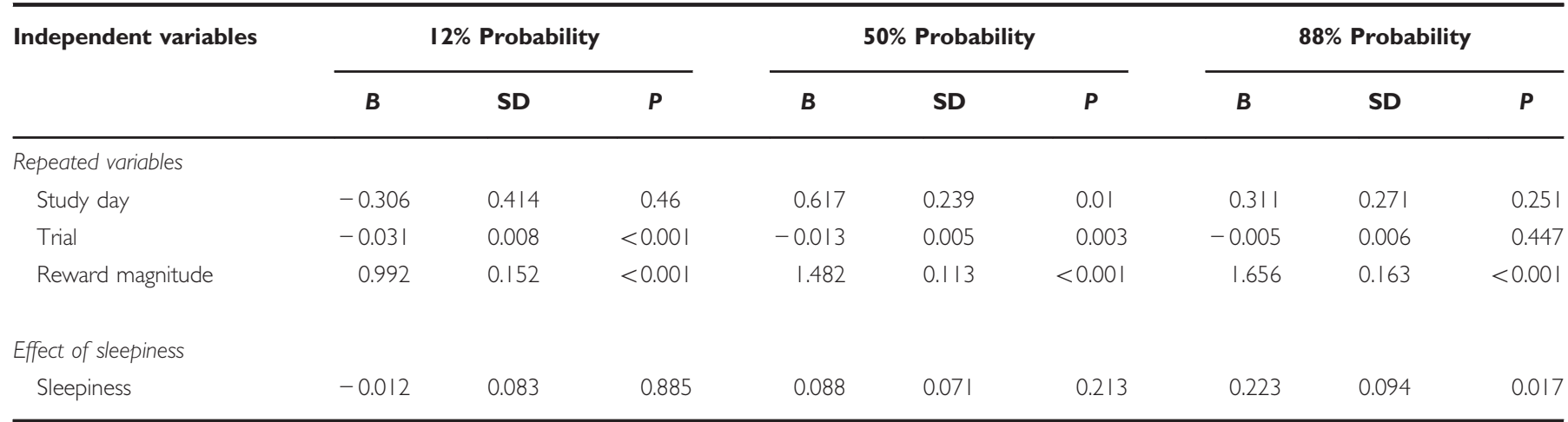

Generalized estimating equation (GEE) using a binary logistic model and high-effort task choice (no/yes) as dependent variable and adjusted for mood state (state anxiety and positive affect) and sickness symptoms (sicknessQ).

whereas the interaction between sleepiness and probability levels remained significant. This result indicates that the observed interaction between LPS administration and probability levels on high-effort task choices was statistically mediated by sleepiness. This indirect effect of sleepiness $x$ probability on the association between LPS $\times$ probability and high-effort task choices was confirmed using the PROCESS macro (indirect effect coefficient: $0.32 ; 95 \%$ confidence interval \pm 0.08$)$. Similar relationships were found when replacing condition by peak IL- 6 concentration as independent variable (see Supplementary Table S2; GEE model 3: peak IL-6 $\times$ probability, $B=0.003(0.003), P=0.193$ and sleepiness $\times$ probability, $B=0.004$ (0.002), $P=0.015$; indirect effect coefficient: $0.17 \pm 0.04$ ).

Notably, the observed associations of LPS administration and sleepiness with the choice of high-effort/high-reward trials were similar when entering the order of the LPS injection (on first $v s$ second study day) as covariate in the model, suggesting no contaminating effect of a potential unblinding (Supplementary Table S3).

\section{Effect of Sleepiness on High-Effort/High-Reward Choices at Each Level of Probability}

Given that a significant and independent interaction was found between sleepiness and probability levels, post hoc analyses were performed to assess the effect of sleepiness at each level of probability $(12,50$, and $88 \%$; Table 2$)$. These analyses revealed that higher levels of sleepiness were associated with increased number of high-effort/high-reward choices, but only when the probability to win was the highest ( $88 \%$; Figure $3 \mathrm{~b}$ ). In other words, when the probability to win was high, individuals with high level of sleepiness made more high-effort/high-reward trial choices than individuals with low levels of sleepiness.

It should be noted that the trial effect observed in the models of the Table 1 was not significant in the high probability level (Table 2), whereas it was significant at the intermediate and low levels of probability to win. This indicates that subjects made less high-effort choices in the course of the task-except when the probability to win was high.

\section{DISCUSSION}

Systemic inflammation induced by LPS administration did not lead to a reduction in incentive motivation in young healthy adults. On the contrary, LPS increased the probability of engaging in high-effort/high-reward trials when the probability to win was high. Further analyses revealed that sleepiness was associated with this effect. Subjects feeling 
sleepy engaged more frequently in the high-effort/highreward trials when the probability to win was maximal. Increase in reward magnitude predicted more high-effort/ high-reward task choices, but independently of the condition (LPS or saline) and sleepiness, meaning that sensitivity to reward remained unaffected.

As mentioned above, after LPS administration, participants chose more frequently the high-effort/high-reward tasks when the probability to win was higher in comparison with saline administration. This effect appeared to be dependent on sleepiness and largely independent of mood alterations and sickness symptoms, given that the models were adjusted for scores of anxiety symptoms, positive affect, and sickness. This finding supports the notion that sleepiness (and probably also fatigue) alters motivation and related behavior, although not in the expected direction. Incentive motivation did not decrease, but rather increased, after exposure to the inflammatory stimulus in relation to sleepiness. This is opposite to previous reports on fatigue in humans who showed a shift of the effort/reward balance so that fatigued subjects are no longer motivated to engage in task performance when energy costs are perceived to outweigh predicted rewards (Boksem and Tops, 2008). The same phenomenon occurs in inflamed rodents (Merali et al, 2003; Nunes et al, 2014). However, most of these studies used high-effort/high-reward modes of response and did not contrast them in the same task with low-effort/low-reward modes of response. Our findings in LPS-treated subjects are actually consistent with the results obtained in LPS-treated mice when tested in a decision-making task (Vichaya et al, 2014). LPS-treated mice increased their proportion of higheffort/high-reward choices despite a decrease in their total number of responses. These findings are in line with the notion that sickness is not associated with a general decrease in motivation but with a reorganization of priorities (Aubert, 1999; Aubert et al, 1997; Larson, 2002). This re-organization of priorities becomes even more apparent in a situation of contrast between different motivational objects where it leads to what has been called 'increased finickiness' (Aubert and Dantzer, 2005). Inflamed subjects are still able to engage in an effort task but are more discerning in their effort allocation. This is illustrated in a recent study in humans, which showed an increased social approach after LPS administration when the target is a close individual (Inagaki et al, 2015), whereas immune activation is known to induce social withdrawal in other conditions (Eisenberger et al, 2010; Inagaki et al, 2012). Likewise, an early report described that rats submitted to forced wheel running and injected with LPS actually increased their effort to press a lever in order to get more rest periods, in comparison with rats injected with saline (Dantzer and Kelley, 2007; Miller, 1964). Behavioral adaptation to contextual cues also appears in fatigued subjects who are able to, at least acutely, overcome the effect of fatigue when the reward is estimated as worthwhile (Boksem et al, 2006; Boksem and Tops, 2008). Altogether, our results are in line with the studies mentioned above, as they indicate that incentive motivation to monetary reward was not reduced but increased in specific conditions, in the context of sickness or fatigue. Our findings show that when the probability to gain the reward is really high, sleepy subjects can still evaluate the cost/benefit balance as most favorable, and are willing to expend more effort to obtain the reward. This is contradictory to the preconceived nature of fatigue and sleepiness; however, it may relate to a need for self-stimulation in the most sleepy subjects when not allowed to sleep. An additional aspect could be that sleepiness may have affected the results via cognitive alterations (Lim and Dinges, 2010) rather than altered motivation.

In the present study, and as hypothesized from animal studies, no difference in the reward sensitivity was observed after LPS $v s$ saline injection or in relation to the sleepiness level. However, a recent study reported a significant reduction in learned reward sensitivity after LPS administration (Harrison et al, 2016). The discrepancy between these findings and the results from the present study may indicate distinct effects depending on whether contrasted rewards are presented (eg, high $v s$ low reward), or may indicate distinct neural representation of the reward depending on whether the pairing between the stimulus and the reward has been made during (ie, in Harrison et al, 2016) or before (eg, during the screening day) the state of sickness, and should be further investigated.

No reduction in task performance was measured after LPS administration. Instead, subjects treated with LPS exhibited increased performance during the high-effort tasks in comparison with subjects treated with saline. In particular, subjects treated with LPS were faster and more successful in the high-effort tasks, perhaps because of compensatory mechanisms involved in counteracting the sickness, or a poorer estimation of the most optimal effort needed to succeed a task. Alternative explanations could be an increased sensitivity/avoidance to negative experiences after inflammatory activation, as it has been shown in rodents with respect to bitter taste (Aubert and Dantzer, 2005) or recently in humans with respect to money loss (Harrison et al, 2016). It is possible that LPS treatment reinforced negative perceptions of the task, resulting in increased irritability and speed of response. Irritability is frequent in sick individuals with systemic inflammation because of, eg, chronic obstructive pulmonary disease or congestive heart failure (Blinderman et al, 2008; Blinderman et al, 2009), but its possible involvement in motivational alterations still needs to be assessed.

A main strength of the study is the experimental randomized within-subject design with 21 subjects using a strong sickness manipulation, giving a reasonably good power to analyze main effects of sickness. The statistical mediation analyses of indirect effects were, however, of cross-sectional nature, and larger samples sizes would be preferred. The EEfRT is a newly developed test to measure motivation, and it has the advantage of being sensitive to several aspects of motivation (Treadway et al, 2012; Treadway et al, 2009; Treadway et al, 2015). However, its sensitivity to non-psychiatric conditions and sleepiness still needs to be confirmed. In addition, the EEfRT only tests for monetary rewards, and the use of a similar task to quantify motivation for social reward (eg, positive $v s$ threatening social cues) rather than a monetary reward would be interesting in order to understand whether the current results are generalizable across different reward natures.

In conclusion, the results of the present study show that systemic inflammation induces incentive motivational changes in young healthy adults that can be objectively assessed using alterations in behavioral performance in a 
monetary reward task. Importantly, the effect was associated with sleepiness and dependent on probability contingencies. We observed that increased sleepiness was related to a higher frequency of high-effort/high-reward choices, but apparently only when the probability to win was high and the effort thus worthwhile. This suggests a complex behavioral pattern during sickness that does not only include energy-saving behaviors in order to promote health-related behaviors, but that allows sick individuals to be able and, sometimes even more inclined, to successfully motivate themselves in higheffort tasks when the effort is appraised as worthwhile. Future studies are needed in order to further comprehend the effects of sickness and related fatigue on motivation.

\section{FUNDING AND DISCLOSURE}

This research was supported by the Swedish Foundation for Humanities and Social Sciences (P12-1017 to MJO), the Swedish Research Council (421-2012-1125) and Stockholm Stress Center. MTT was supported by the National Institute of Mental Health (R00MH102355). In the last 3 years, MTT has consulted for the Boston Consulting Group, Avanir Pharmaceuticals and NeuroCog. No funding or sponsorship was provided by these companies for the current work, and all views expressed herein are solely those of authors. MTT has also received honoraria and royalties related to his writing. The authors declare no conflict of interest.

\section{ACKNOWLEDGMENTS}

We thank Professor H Wallén and Dr L Hållström for their help in designing the protocol and for their technical and medical support. We thank L Gabrielsson and I Hellström for help with data collection.

\section{REFERENCES}

Akerstedt T, Anund A, Axelsson J, Kecklund G (2014). Subjective sleepiness is a sensitive indicator of insufficient sleep and impaired waking function. J Sleep Res 23: 240-252.

Andreasson A, Wicksell RK, Lodin K, Karshikoff B, Axelsson J, Lekander M (2016). A global measure of sickness behavior: development of the sickness questionnaire (SicknessQ). Journal of Health Psychology (doi:10.1177/1359105316659917).

Anisman H, Kokkinidis L, Borowski T, Merali Z (1998). Differential effects of interleukin (IL)-1beta, IL-2 and IL-6 on responding for rewarding lateral hypothalamic stimulation. Brain Res 779: $177-187$.

Aubert A (1999). Sickness and behaviour in animals: a motivational perspective. Neurosci Biobehav Rev 23: 1029-1036.

Aubert A, Dantzer R (2005). The taste of sickness: lipopolysaccharide-induced finickiness in rats. Physiol Behav 84: 437-444.

Aubert A, Goodall G, Dantzer R, Gheusi G (1997). Differential effects of lipopolysaccharide on pup retrieving and nest building in lactating mice. Brain Behav Immun 11: 107-118.

Berridge KC, Robinson TE, Aldridge JW (2009). Dissecting components of reward: 'liking', 'wanting', and learning. Curr Opin Pharmacol 9: 65-73.

Blinderman CD, Homel P, Billings JA, Portenoy RK, Tennstedt SL (2008). Symptom distress and quality of life in patients with advanced congestive heart failure. J Pain Symptom Manage 35: 594-603.
Blinderman CD, Homel P, Billings JA, Tennstedt S, Portenoy RK (2009). Symptom distress and quality of life in patients with advanced chronic obstructive pulmonary disease. J Pain Symptom Manage 38: 115-123.

Boksem MA, Meijman TF, Lorist MM (2006). Mental fatigue, motivation and action monitoring. Biol Psychol 72: 123-132.

Boksem MA, Tops M (2008). Mental fatigue: costs and benefits. Brain Res Rev 59: 125-139.

Calvano SE, Coyle SM (2012). Experimental human endotoxemia: a model of the systemic inflammatory response syndrome? Surg Infect (Larchmt) 13: 293-299.

Capuron L, Pagnoni G, Drake DF, Woolwine BJ, Spivey JR, Crowe RJ et al (2012). Dopaminergic mechanisms of reduced basal ganglia responses to hedonic reward during interferon alfa administration. Arch Gen Psychiatry 69: 1044-1053.

Dantzer R, Heijnen CJ, Kavelaars A, Laye S, Capuron L (2014). The neuroimmune basis of fatigue. Trends Neurosci 37: 39-46.

Dantzer R, Kelley KW (2007). Twenty years of research on cytokine-induced sickness behavior. Brain Behav Immun 21: 153-160.

Dantzer R, O'Connor JC, Freund GG, Johnson RW, Kelley KW (2008). From inflammation to sickness and depression: when the immune system subjugates the brain. Nat Rev Neurosci 9: $46-56$.

De La Garza R 2nd, Asnis GM, Fabrizio KR, Pedrosa E (2005). Acute diclofenac treatment attenuates lipopolysaccharide-induced alterations to basic reward behavior and HPA axis activation in rats. Psychopharmacology 179: 356-365.

Eisenberger NI, Berkman ET, Inagaki TK, Rameson LT, Mashal NM, Irwin MR (2010). Inflammation-induced anhedonia: endotoxin reduces ventral striatum responses to reward. Biol Psychiatry 68: 748-754.

Engler H, Benson S, Wegner A, Spreitzer I, Schedlowski M, Elsenbruch S (2016). Men and women differ in inflammatory and neuroendocrine responses to endotoxin but not in the severity of sickness symptoms. Brain Behav Immun 52: 18-26.

Felger JC, Miller AH (2012). Cytokine effects on the basal ganglia and dopamine function: the subcortical source of inflammatory malaise. Front Neuroendocrinol 33: 315-327.

Hallstrom L, Berghall E, Frostell C, Sollevi A, Soop AL (2011). Immunomodulation by a combination of nitric oxide and glucocorticoids in a human endotoxin model. Acta Anaesthesiol Scand 55: 20-27.

Harrison NA, Brydon L, Walker C, Gray MA, Steptoe A, Dolan RJ et al (2009). Neural origins of human sickness in interoceptive responses to inflammation. Biol Psychiatry 66: 415-422.

Harrison NA, Voon V, Cercignani M, Cooper EA, Pessiglione M, Critchley HD (2016). A neurocomputational account of how inflammation enhances sensitivity to punishments versus rewards. Biol Psychiatry 80: 73-81.

Hayes AF (2013). Introduction to Mediation, Moderation, and Conditional Process Analysis: a Regression-Based Approach. The Guilford Press: New York, NY, USA.

Hermann DM, Mullington J, Hinze-Selch D, Schreiber W, Galanos C, Pollmacher T (1998). Endotoxin-induced changes in sleep and sleepiness during the day. Psychoneuroendocrinology 23: 427-437.

Inagaki TK, Muscatell KA, Irwin MR, Cole SW, Eisenberger NI (2012). Inflammation selectively enhances amygdala activity to socially threatening images. Neuroimage 59: 3222-3226.

Inagaki TK, Muscatell KA, Irwin MR, Moieni M, Dutcher JM, Jevtic I et al (2015). The role of the ventral striatum in inflammatory-induced approach toward support figures. Brain Behav Immun 44: 247-252.

Karshikoff B, Lekander M, Soop A, Lindstedt F, Ingvar M, Kosek E, Olgart Hoglund C, Axelsson J (2015). Modality and sex differences in pain sensitivity during human endotoxemia. Brain Behav Immun 46: 35-43. 
Larson SJ (2002). Behavioral and motivational effects of immunesystem activation. J Gen Psychol 129: 401-414.

Lasselin J, Elsenbruch S, Lekander M, Axelsson J, Karshikoff B, Grigoleit JS et al (2016). Mood disturbance during experimental endotoxemia: predictors of state anxiety as a psychological component of sickness behavior. Brain Behav Immun 57: 30-37.

Lim J, Dinges DF (2010). A meta-analysis of the impact of shortterm sleep deprivation on cognitive variables. Psychol Bull 136: 375-389.

Merali Z, Brennan K, Brau P, Anisman H (2003). Dissociating anorexia and anhedonia elicited by interleukin-1beta: antidepressant and gender effects on responding for 'free chow' and 'earned' sucrose intake. Psychopharmacology 165: 413-418.

Miller AH, Raison CL (2015). The role of inflammation in depression: from evolutionary imperative to modern treatment target. Nat Rev Immunol 16: 22-34.

Miller NE (1964). Some psychophysiological studies of motivation and of the behavioural effects of illness. Bull Br Psychol Soc 17: $1-20$.

Nunes EJ, Randall PA, Estrada A, Epling B, Hart EE, Lee CA et al (2014). Effort-related motivational effects of the proinflammatory cytokine interleukin 1-beta: studies with the concurrent fixed ratio 5/ chow feeding choice task. Psychopharmacology 231: 727-736.

Salamone JD, Correa M, Farrar A, Mingote SM (2007). Effortrelated functions of nucleus accumbens dopamine and associated forebrain circuits. Psychopharmacology 191: 461-482.

Sheehan DV, Lecrubier Y, Sheehan KH, Amorim P, Janavs J, Weiller E et al (1998). The Mini-International Neuropsychiatric
Interview (M.I.N.I.): the development and validation of a structured diagnostic psychiatric interview for DSM-IV and ICD-10. J Clin Psychiatry 59 Suppl 20: 22-33.

Spielberger CD, Gorsuch RL, Lushene R (1979). Manual of the State-Trait Anxiety Inventory. Consulting Psychologists Press: Palo Alto, CA, USA.

Suffredini AF, Hochstein HD, McMahon FG (1999). Dose-related inflammatory effects of intravenous endotoxin in humans: evaluation of a new clinical lot of Escherichia coli O:113 endotoxin. J Infect Dis 179: 1278-1282.

Treadway MT, Bossaller NA, Shelton RC, Zald DH (2012). Effortbased decision-making in major depressive disorder: a translational model of motivational anhedonia. J Abnorm Psychol 121: 553-558.

Treadway MT, Buckholtz JW, Schwartzman AN, Lambert WE, Zald DH (2009). Worth the 'EEfRT'? The effort expenditure for rewards task as an objective measure of motivation and anhedonia. PLoS One 4: e6598.

Treadway MT, Peterman JS, Zald DH, Park S (2015). Impaired effort allocation in patients with schizophrenia. Schizophr Res 161: 382-385.

Vastfjall D, Garling T (2007). Validation of a Swedish short selfreport measure of core affect. Scand J Psychol 48: 233-238.

Vichaya EG, Hunt SC, Dantzer R (2014). Lipopolysaccharide reduces incentive motivation while boosting preference for high reward in mice. Neuropsychopharmacology 39: 2884-2890.

Wardle MC, Treadway MT, Mayo LM, Zald DH, de Wit H (2011). Amping up effort: effects of d-amphetamine on human effortbased decision-making. J Neurosci 31: 16597-16602.

Supplementary Information accompanies the paper on the Neuropsychopharmacology website (http://www.nature.com/npp) 